\title{
MAINTENANCE AND PRODUCING OF THE NUCLEOLUS (BREEDER'S SEED) OF GIZA 92 EGYPTIAN COTTON CULTIVAR (GOSSYPIUM BARBADENSE L)
}

\author{
M.A.A. El-Dahan \\ Cotton Research Institute, Agricultural Research Center, Giza, Egypt. \\ (E-mail: ashour6es@yahoo.es)
}

\begin{abstract}
The breeding program of the cultivar Giza 92 was conducted at Sakha Agricultural Research Station, Kafr El-Sheikh district during 2013 -2016 growing seasons to initiate a nucleolus. In 2013 season thirty one type plants were selected from the breeding field of Giza 92 cultivar and provided thirty one progenies (increase A) in 2014. From increase A, seventeen families were selected to establish increase B in 2015. According to the statistical analysis of yield trial which included the seventeen families and comparisons of the latest three lines in cultivation of Giza 92, Seven elite families were selected and the seeds were carefully massed together to form the nucleolus (breeder's seed) in 2016 season. The results obtained indicated that, the pure line method in the sense of pedigree selection for renewing annually Giza 92 breeder's seed could produce high genetically pure seeds and meantime, prevent genetic deterioration. Meanwhile, the selection technique for producing breeder's seed of the variety was valid and proved to be effective in holding this variety according to the standard type of Giza 92.
\end{abstract}

Key words: Maintenance, Cotton, Giza 92

INTRODUCTION

Egyptian cotton is considered a distinctive type of cotton that is characterized by high quality, and gained a world-wide reputation as being of the highest lint quality among world cottons. Its fineness, strength and superior characteristics, have positioned its products as the world's finest. This reputation in the course of time was attributed to the maintenance procedure followed by Department of Varietal Maintenance in Cotton Research Institute, to maintain the genetic purity and identity standard characteristics of Egyptian cotton varieties. Pedigree selection method has become the most common plant breeding procedure. All Egyptian cotton varieties are maintained by this method. Both of pedigree selection and independent culling levels were used in maintenance and renewing Egyptian cotton varieties. Lewis (1970) indicated that Egyptian cotton varietal maintenance consider essential in breeding program to maintain high quality properties and prevent any deterioration for these traits. Maintenance 
of Egyptian cotton varieties have been reported by many researchers Ware (1959), Turner (1963), Walker (1964) and Riggs (1967), they studied the bulk model system designed to stabilize a variety. They concluded that this system could be considered for cotton variety maintenance. Al-Didi (1974) stated that it was advantageous to mass the seed of the chosen families in which the seed mixture may respond differently to environmental variation and if genotype $x$ environment effects were significant, mixture of seeds might show less fluctuation in yield and quality than individual progenies However, El-Akkad et. al., (1982), El-Kilany and Yousef (1985), Younis et. al., (1993), Lasheen (1997) and Al-Ameer (2014) reported that the pure seed and production of cotton cultivar using pedigree selection method is essential to produce renew and maintain the breeder's seed of the cotton cultivars in the commercial use. This method based on massing selfed seeds of homogeneous type of families, according to their performance in evaluation with the latest nuclei. Cotton Varietal Maintenance Department is the responsible of maintaining and renewing breeder's seed of the commercial cultivars and the further seed production steps are carried out with the collaboration with Central Administration for Seed Production and Central Administration for Seed Certification. The main objective of this work is to follow the procedure of renewing and maintaining to produce pure breeder's seed of Giza 92.

\section{MATERIALS AND METHODS}

Giza 92 cotton variety is a commercial Egyptian cotton cultivar (G. barabadense L.) cultivated at north delta region and classified extra-long stable with a stable length $(34.5-35 \mathrm{~mm})$ and represents the highest level of fiber strength in the world (50-53 g/tex). This cultivar was derived by the pedigree selection method from the cross between Giza 84 x (Giza 74 x Giza 68) and released commercially in 2011. The present study was carried out at Sakha Agricultural Research Station, Cotton Res., Inst., during four seasons from 2013 to 2016.

The basic materials for this study were the individual elite plants selected based on field evaluation and laboratory determination from breeding plot of 2012 season. At harvest each selected individual plant in the breeding plot was picked separately. The plants were screened for yield, yield components as well as fiber properties. In 2013 season, thirty-one plants representing the type of Giza 92 cultivar were selected to form the increase lines $A$.

In 2014 season the selfed seeds of the progenies of the 31 selected type plants were grown in number of rows as the amount of seed allowed conveniently named increase line $A$, as well as the natural pollinated seeds of same 31 selected type plants were grown 
adjacent progeny three rows to be increased for using it in yield trial in the next year. According to the field and laboratory tests of phenotypic yield and its components and fiber properties, 17 families were selected from increase A. In 2015 season, the selefed seeds of the 17 families were grown in increase B. A yield trial included the 17 selected families as well as the three latest strains of Giza 92 were used as controls. The design of yield trail was a randomized complete blocks design with four replications. The 17 selected families as well as the three latest control strains of G 92 were evaluated for yield, its components and fiber properties. In 2016 season, according to the results of yield trail, the best seven families representing the type of Giza 92 cultivar were selected from increase B and their selfed seeds were carefully massed together to form the new nucleolus (breeder's seed) and propagated in 2016 under the name of season (Giza 92 nucleolus/2016) in about 10 feddans at Sakha farm.

Data of the following traits were recorded:

Yield and yield component traits are:

1. Seed cotton yield (SCY) in K/Fed.

2. Lint cotton yield (LCY) in K/Fed.

3. Lint percentage $(\mathrm{L} \%)$.

4. Boll weight (BW) in $\mathrm{g}$.

5. Seed index (SI) in $\mathrm{g}$.

6. Lint index (LI) in $\mathrm{g}$.

Fiber properties:

1. Fiber length at $2.5 \%$ Span length $(2.5 \% \mathrm{SL})$ in $\mathrm{mm}$.

2. Uniformity index (UI \%).

3. Fiber strength; Pressley index (PI), strength (ST) (g/tex) and yarn strength (YS).

4. Elongation ( $\mathrm{E} \%$ ).

5. Micronaire value (MV).

6. Maturity ratio (MR)

7. Brightness (Rd \%).

8. Yellowness $(+b)$.

Mean of the selected families and comparison, standard error and coefficient of variability (CV \%) were calculated for all the studied traits, also analysis of variance were carried out for all the studied traits in the yield trail.

\section{Ideal type plants in 2013 season:}

\section{RESULTS AND DISCUSSION}

Mean of yield component characters and fiber properties for the selected 31 type plants of Giza 92 cultivar in 2013 season, are shown 
in Table 1. Small values of SE indicate that the sample means is more accurate reflecting the actual Giza 92 population mean. Whereas, coefficient of variability were low for L\%., $205 \% 2.5 \%$ SL and pressley index indicating less dispersion for these traits, whoever coefficient of variability were relatively high for $\mathrm{BW}, \mathrm{SI}, \mathrm{LI}$ and MV, this could be due to environmental effects on these traits. Similar results were obtained by Abo-Arab et. al., (1995). El-Disouqi (2001), Abd Alzaher (2004) and Mohamed (2013).

The relative lower values of standard error (SE) and the coefficient of variability (CV \%) indicate the high homogeneity between the selected type plants. These results indicated that the selected characters are demonstrating the standard characteristics of Giza 92 cultivar and its behavior across generations.

Table 1: Means of yield components and fiber properties of the 31 selected type plants of Giza 92 from 2013 season that will form the increase A in 2014 season

\begin{tabular}{|c|c|c|c|c|c|c|c|c|}
\hline ì & 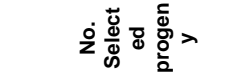 & $\sum_{0}^{01}$ & $\stackrel{\circ}{~}$ & ๘ & $\frac{\sigma}{\square}$ & $\begin{array}{l}\text { ㅇ E } \\
\text { மீ } \frac{1}{\omega}\end{array}$ & $\bar{\alpha}$ & $\grave{\Sigma}$ \\
\hline 1 & $2 / 2013-4$ & 3.1 & 34.4 & 9.6 & 5.0 & 35.1 & 11.0 & 3.5 \\
\hline 2 & $2 / 2013-30$ & 3.0 & 35.0 & 9.5 & 5.1 & 35.2 & 11.2 & 3.6 \\
\hline 3 & $5 / 2013-8$ & 3.1 & 36.3 & 9.3 & 5.3 & 37.7 & 11.0 & 3.8 \\
\hline 4 & $5 / 2013-11$ & 3.2 & 36.3 & 9.8 & 5.6 & 37.7 & 12.5 & 3.8 \\
\hline 5 & $24 / 2013-4$ & 3.3 & 35.9 & 9.3 & 5.2 & 34.9 & 11.6 & 3.5 \\
\hline 6 & $26 / 2013-4$ & 3.6 & 36.1 & 10.6 & 6.0 & 35.6 & 11.0 & 3.2 \\
\hline 7 & $26 / 2013-23$ & 3.4 & 37.1 & 10.3 & 6.1 & 34.9 & 11.4 & 3.4 \\
\hline 8 & $26 / 2013-30$ & 3.6 & 35.2 & 9.7 & 5.3 & 35.2 & 11.5 & 3.5 \\
\hline 9 & $26 / 2013-31$ & 3.4 & 36.4 & 10.0 & 5.7 & 34.8 & 11.3 & 2.9 \\
\hline 10 & $27 / 2013-29$ & 3.6 & 35.2 & 10.9 & 5.9 & 34.7 & 11.2 & 3.8 \\
\hline 11 & $27 / 2013-40$ & 3.7 & 35.2 & 10.0 & 5.4 & 35.5 & 11.1 & 3.7 \\
\hline 12 & $38 / 2013-17$ & 3.3 & 35.6 & 10.3 & 5.7 & 35.1 & 11.4 & 3.7 \\
\hline 13 & $38 / 2013-21$ & 3.5 & 36.5 & 10.5 & 6.0 & 34.6 & 11.2 & 3.9 \\
\hline 14 & $41 / 2013-8$ & 3.3 & 35.9 & 9.6 & 5.4 & 35.7 & 11.8 & 3.6 \\
\hline 15 & $43 / 2013-2$ & 3.6 & 35.8 & 10.2 & 5.7 & 35.1 & 11.3 & 3.7 \\
\hline 16 & $43 / 2013-8$ & 3.7 & 35.5 & 9.7 & 5.3 & 35.7 & 11.0 & 3.5 \\
\hline 17 & $44 / 2013-21$ & 3.5 & 35.1 & 11.8 & 6.4 & 35.6 & 11.3 & 3.6 \\
\hline 18 & $46 / 2013-15$ & 3.6 & 36.7 & 9.9 & 5.7 & 34.7 & 11.4 & 3.8 \\
\hline 19 & $52 / 2013-9$ & 3.8 & 35.6 & 10.6 & 5.9 & 35.5 & 11.2 & 3.4 \\
\hline 20 & $52 / 2013-27$ & 3.8 & 36.6 & 10.9 & 6.3 & 34.6 & 11.0 & 3.4 \\
\hline 21 & $52 / 2013-31$ & 3.9 & 35.7 & 10.0 & 5.6 & 35.5 & 11.3 & 3.5 \\
\hline 22 & $53 / 2013-6$ & 3.5 & 36.3 & 11.5 & 6.6 & 34.6 & 11.0 & 3.2 \\
\hline 23 & $53 / 2013-8$ & 3.4 & 35.3 & 10.5 & 5.7 & 34.6 & 11.2 & 3.3 \\
\hline 24 & $54 / 2013-16$ & 3.4 & 35.5 & 11.5 & 6.3 & 35.0 & 11.2 & 3.3 \\
\hline 25 & $54 / 2013-17$ & 3.3 & 35.5 & 11.3 & 6.2 & 34.9 & 11.2 & 3.6 \\
\hline 26 & $54 / 2013-20$ & 3.3 & 35.6 & 10.9 & 6.0 & 35.2 & 11.7 & 3.3 \\
\hline 27 & $55 / 2013-21$ & 3.9 & 36.5 & 9.9 & 5.7 & 36.3 & 11.5 & 3.5 \\
\hline 28 & $57 / 2013-9$ & 3.3 & 36.4 & 9.5 & 5.4 & 35.6 & 11.2 & 3.5 \\
\hline 29 & $58 / 2013-25$ & 3.1 & 35.2 & 10.4 & 5.6 & 34.8 & 11.4 & 3.5 \\
\hline 30 & $60 / 2013-5$ & 3.2 & 36.3 & 10.3 & 5.9 & 36.0 & 11.0 & 3.5 \\
\hline 31 & $60 / 2013-25$ & 3.5 & 35.4 & 10.0 & 5.5 & 34.6 & 11.2 & 3.8 \\
\hline \multicolumn{2}{|c|}{ Mean of selected progenies } & 3.5 & 35.8 & 10.3 & 5.7 & 35.3 & 11.3 & 3.5 \\
\hline \multicolumn{2}{|c|}{ Mean of comparisons (control) } & 3.3 & 35.7 & 10.0 & 5.6 & 34.5 & 10.4 & 3.5 \\
\hline \multicolumn{2}{|c|}{ SE } & 0.043 & 0.110 & 0.120 & 0.071 & 0.139 & 0.055 & 0.039 \\
\hline \multicolumn{2}{|c|}{ CV \% } & 6.98 & 1.71 & 6.50 & 6.93 & 2.20 & 2.71 & 6.21 \\
\hline
\end{tabular}

$\mathrm{SE}=$ Standard Error 


\section{Increase A (2014 season)}

Mean values of yield components and fiber properties of the 31 families were compared with the three latest commercial strains of Giza 92. The results are presented in Table 2. It could be noticed that, the mean of progenies (increase A) slightly differed from the mean comparisons for most traits. Also SE and CV values were relatively low for all traits. These results indicate gene fixation and homogeneity of the studied Giza 92 families.

Table 2: Means of yield components and fiber properties for the 31 selected progeny (increase A) in 2014 growing season

\begin{tabular}{|c|c|c|c|c|c|c|c|c|c|c|c|c|c|}
\hline ì & 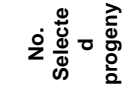 & $\sum_{0}^{\sigma}$ & $\stackrel{\circ}{\circ}$ & ๘ & $\frac{\sigma}{\square}$ & 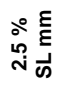 & $\frac{\circ}{5}$ & ๒ & $\stackrel{\infty}{\nu}$ & $\mathbf{\Sigma}$ & ஷ̊ํ & $\stackrel{0}{+}$ & $\stackrel{\circ}{\circ}$ \\
\hline 1 & $2 / 2013-4$ & 3.32 & 37.5 & 11.5 & 6.9 & 34.7 & 87.7 & 50.2 & 2880 & 3.9 & 7.4 & 7.7 & 80.5 \\
\hline 2 & $2 / 2013-30$ & 3.04 & 37.5 & 12.0 & 7.2 & 33.7 & 87.3 & 48.3 & 2795 & 3.7 & 7.7 & 7.9 & 80.8 \\
\hline 3 & $5 / 2013-8$ & 3.30 & 37.5 & 11.3 & 6.8 & 35.2 & 87.7 & 49.3 & 2995 & 3.8 & 7.3 & 8.0 & 80.5 \\
\hline 4 & $5 / 2013-11$ & 3.14 & 37.8 & 11.4 & 6.9 & 34.3 & 87.8 & 46.5 & 2770 & 3.7 & 7.0 & 8.4 & 82.3 \\
\hline 5 & $24 / 2013-4$ & 3.02 & 38.0 & 10.5 & 6.4 & 33.7 & 86.5 & 53.3 & 2590 & 3.8 & 7.5 & 8.0 & 82.1 \\
\hline 6 & $26 / 2013-4$ & 3.06 & 38.0 & 11.1 & 6.8 & 35.3 & 88.4 & 47.3 & 2825 & 3.7 & 7.8 & 8.3 & 82.0 \\
\hline 7 & $26 / 2013-23$ & 3.02 & 38.0 & 11.9 & 7.3 & 36.1 & 95.2 & 46.5 & 2785 & 3.9 & 7.0 & 8.2 & 80.9 \\
\hline 8 & $26 / 2013-30$ & 3.08 & 38.0 & 10.8 & 6.6 & 35.8 & 90.2 & 45.0 & 2820 & 3.7 & 7.2 & 7.5 & 79.0 \\
\hline 9 & $26 / 2013-31$ & 3.05 & 38.5 & 10.9 & 6.8 & 35.4 & 90.2 & 43.4 & 2520 & 3.7 & 7.1 & 8.1 & 81.6 \\
\hline 10 & $27 / 2013-29$ & 3.12 & 38.5 & 10.1 & 6.3 & 34.3 & 87.3 & 40.2 & 2500 & 3.7 & 7.8 & 7.8 & 80.5 \\
\hline 11 & $27 / 2013-40$ & 3.10 & 38.5 & 10.8 & 6.7 & 34.0 & 86.9 & 45.2 & 2765 & 3.9 & 7.8 & 8.6 & 80.1 \\
\hline 12 & $38 / 2013-17$ & 3.11 & 39.0 & 10.1 & 6.5 & 34.5 & 86.2 & 46.5 & 2790 & 3.8 & 7.6 & 8.0 & 81.7 \\
\hline 13 & $38 / 2013-21$ & 3.06 & 38.5 & 10.3 & 6.4 & 32.9 & 84.7 & 46.3 & 2700 & 4.0 & 7.8 & 8.1 & 81.7 \\
\hline 14 & $41 / 2013-8$ & 3.08 & 38.0 & 10.7 & 6.6 & 34.5 & 89.5 & 47.1 & 2770 & 4.0 & 7.5 & 8.4 & 80.4 \\
\hline 15 & $43 / 2013-2$ & 3.08 & 38.0 & 10.2 & 6.2 & 33.8 & 87.8 & 44.5 & 2580 & 3.8 & 7.6 & 8.3 & 81.0 \\
\hline 16 & $43 / 2013-8$ & 3.88 & 36.3 & 10.5 & 6.0 & 35.3 & 89.2 & 47.0 & 2825 & 4.0 & 7.7 & 8.1 & 80.3 \\
\hline 17 & $44 / 2013-21$ & 3.45 & 37.0 & 11.5 & 6.8 & 34.3 & 89.0 & 45.8 & 2685 & 4.0 & 7.5 & 8.4 & 81.3 \\
\hline 18 & $46 / 2013-15$ & 3.27 & 37.0 & 11.9 & 7.0 & 34.5 & 84.6 & 47.9 & 2670 & 3.9 & 7.8 & 8.1 & 79.5 \\
\hline 19 & $52 / 2013-9$ & 3.12 & 39.0 & 11.5 & 7.3 & 35.1 & 85.6 & 44.4 & 2790 & 3.9 & 7.7 & 8.3 & 80.7 \\
\hline 20 & $52 / 2013-27$ & 3.08 & 38.5 & 11.4 & 7.1 & 36.1 & 90.0 & 50.3 & 2940 & 4.0 & 7.2 & 8.7 & 80.0 \\
\hline 21 & $52 / 2013-31$ & 3.31 & 38.5 & 11.0 & 6.9 & 35.3 & 88.5 & 46.5 & 2800 & 4.0 & 7.9 & 7.5 & 79.5 \\
\hline 22 & $53 / 2013-6$ & 3.07 & 39.0 & 11.1 & 7.1 & 34.4 & 88.7 & 48.3 & 2720 & 3.8 & 7.3 & 8.1 & 81.5 \\
\hline 23 & $53 / 2013-8$ & 3.19 & 38.8 & 10.2 & 6.5 & 33.5 & 83.6 & 45.8 & 2495 & 3.9 & 7.9 & 8.2 & 81.8 \\
\hline 24 & $54 / 2013-16$ & 3.09 & 38.8 & 11.0 & 7.0 & 35.2 & 85.3 & 50.0 & 2880 & 3.6 & 7.7 & 7.7 & 80.5 \\
\hline 25 & $54 / 2013-17$ & 3.14 & 38.5 & 11.2 & 7.0 & 33.7 & 85.2 & 50.5 & 2745 & 3.8 & 7.2 & 8.2 & 80.3 \\
\hline 26 & $54 / 2013-20$ & 3.30 & 39.3 & 10.6 & 6.8 & 36.4 & 90.4 & 47.6 & 2730 & 3.8 & 7.8 & 8.0 & 80.3 \\
\hline 27 & $55 / 2013-21$ & 3.05 & 40.0 & 10.1 & 6.7 & 35.3 & 82.4 & 47.4 & 2620 & 3.8 & 7.2 & 7.3 & 77.8 \\
\hline 28 & $57 / 2013-9$ & 3.19 & 38.5 & 10.5 & 6.5 & 34.5 & 85.9 & 46.5 & 2790 & 3.5 & 7.3 & 7.9 & 79.7 \\
\hline 29 & $58 / 2013-25$ & 3.19 & 40.0 & 10.5 & 7.0 & 33.4 & 83.5 & 47.0 & 2590 & 3.6 & 7.8 & 8.2 & 80.2 \\
\hline 30 & $60 / 2013-5$ & 3.25 & 39.0 & 11.0 & 7.0 & 33.3 & 84.9 & 48.3 & 2680 & 3.6 & 7.7 & 8.1 & 78.2 \\
\hline 31 & $60 / 2013-25$ & 3.25 & 38.3 & 10.0 & 6.2 & 34.4 & 85.5 & 44.3 & 2545 & 3.6 & 7.2 & 8.4 & 79.7 \\
\hline \multicolumn{2}{|c|}{$\begin{array}{l}\text { Mean of selected } \\
\text { progenies }\end{array}$} & 3.17 & 38.3 & 10.9 & 6.75 & 34.6 & 87.3 & 47.0 & 2728 & 3.80 & 7.52 & 8.08 & 80.5 \\
\hline \multicolumn{2}{|c|}{$\begin{array}{c}\text { Mean of Comparisons } \\
\text { (control) }\end{array}$} & 3.22 & 39.3 & 10.8 & 7.00 & 34.4 & 87.9 & 47.7 & 2723 & 3.73 & 7.10 & 7.97 & 79.5 \\
\hline \multicolumn{2}{|c|}{ SE } & 0.03 & 0.14 & 0.10 & 0.06 & 0.16 & 0.47 & 0.45 & 22.8 & 0.03 & 0.05 & 0.06 & 0.19 \\
\hline \multicolumn{2}{|c|}{ CV \% } & 5.34 & 2.11 & 5.30 & 4.91 & 2.57 & 2.98 & 5.29 & 4.65 & 3.75 & 3.76 & 3.92 & 1.34 \\
\hline
\end{tabular}

Application of independent culling levels selection for increase A, revealed 17 families were selected according to Giza 92 standard characteristics to form increase B families, these families were compared in yield trail in 2015 season. 


\section{Increase B (2015 season)}

The 17 families selected in 2014 season were grown in 2015 season in number of rows according to seed quantity. These families were evaluated in yield trail with the latest three strains; Table 3 showed the mean values of these families for yield, yield components and fiber properties, analysis of variance for yield and yield components showed there were no significant differences between these families for these traits ( $P$ value $>0.05$ ), also $S E$ and $C V$ were relatively low for all the studied traits except CV for SCY and LCY, were relatively high indicating the homogeneity among all families in increase (B), these results agree with those obtained by Abdel-Al (1976), Abo-Arab et al., (1995), Lasheen (1997), El-Disoqui (2001) Nagib and Hemida (2001), Abd Al-Zaher (2004), Mohamed (2013) and Al-Ameer (2014).

Table 3: Means of yield, yield components and fiber properties of the 17 selected families (increase B) in 2015 growing season furnishing nucleolus in 2016 season

\begin{tabular}{|c|c|c|c|c|c|c|c|c|c|c|c|c|c|c|c|}
\hline i & 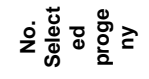 & ত্ৰ & 己ั & $\sum_{0}^{\infty}$ & $\stackrel{\circ}{\circ}$ & ๘ & $\stackrel{\sigma}{\Xi}$ & $\begin{array}{l}\stackrel{\circ}{\circ} \\
\stackrel{\operatorname{Ln}}{\text { ஸे }}\end{array}$ & $\frac{\circ}{5}$ & 心 & $\mathbf{\Sigma}$ & ஷ̊ํ & $\stackrel{0}{+}$ & $\stackrel{\mathfrak{q}}{\Sigma}$ & 。̊ \\
\hline 1 & $2 / 2013-4$ & 6.2 & 7.2 & 2.8 & 36.8 & 11.5 & 6.7 & 33.9 & 87.0 & 48.5 & 3.5 & 6.3 & 8.3 & 0.93 & 77.3 \\
\hline 2 & $5 / 2013-8$ & 5.8 & 6.4 & 2.7 & 35.8 & 11.4 & 6.4 & 35.5 & 87.5 & 48.1 & 3.6 & 6.3 & 8.7 & 0.92 & 78.9 \\
\hline 3 & 5/2013-11 & 7.1 & 7.9 & 2.8 & 35.6 & 11.4 & 6.3 & 34.0 & 87.1 & 50.1 & 3.4 & 6.7 & 8.7 & 0.92 & 77.6 \\
\hline 4 & $26 / 2013-4$ & 5.9 & 6.8 & 2.8 & 36.1 & 11.2 & 6.4 & 33.2 & 84.7 & 47.9 & 3.3 & 6.4 & 8.5 & 0.89 & 78.3 \\
\hline 5 & $26 / 2013-23$ & 6.9 & 7.9 & 3.0 & 36.6 & 11.0 & 6.4 & 36.8 & 86.3 & 45.9 & 3.5 & 6.3 & 8.5 & 0.90 & 77.1 \\
\hline 6 & $26 / 2013-30$ & 8.5 & 9.6 & 2.8 & 35.9 & 11.2 & 6.3 & 33.4 & 85.2 & 47.6 & 3.7 & 6.1 & 8.5 & 0.93 & 78.4 \\
\hline 7 & $38 / 2013-17$ & 8.2 & 9.5 & 2.9 & 36.9 & 10.8 & 6.3 & 35.2 & 87.2 & 45.3 & 3.5 & 6.8 & 8.5 & 0.91 & 76.5 \\
\hline 8 & $41 / 2013-8$ & 8.7 & 10.0 & 3.0 & 36.6 & 11.0 & 6.3 & 33.5 & 85.3 & 45.9 & 3.6 & 6.5 & 8.6 & 0.90 & 77.1 \\
\hline 9 & $43 / 2013-8$ & 6.1 & 6.8 & 2.8 & 35.0 & 10.9 & 5.9 & 34.0 & 82.8 & 44.5 & 3.6 & 6.8 & 9.1 & 0.90 & 77.3 \\
\hline 10 & $46 / 2013-15$ & 6.3 & 7.0 & 2.8 & 35.8 & 10.8 & 6.0 & 34.5 & 84.6 & 47.9 & 3.9 & 7.8 & 8.1 & 0.92 & 79.5 \\
\hline 11 & $52 / 2013-27$ & 7.0 & 8.1 & 2.8 & 36.7 & 10.9 & 6.3 & 33.7 & 84.5 & 49.0 & 3.6 & 6.0 & 8.4 & 0.94 & 75.6 \\
\hline 12 & $52 / 2013-31$ & 6.9 & 8.0 & 2.8 & 36.5 & 11.0 & 6.3 & 34.0 & 83.3 & 46.5 & 3.5 & 6.8 & 8.1 & 0.91 & 77.2 \\
\hline 13 & $53 / 2013-6$ & 7.7 & 8.8 & 2.9 & 36.3 & 10.7 & 6.1 & 34.2 & 89.0 & 49.6 & 3.5 & 6.6 & 8.1 & 0.93 & 76.7 \\
\hline 14 & $54 / 2013-16$ & 7.1 & 8.4 & 3.0 & 37.3 & 10.7 & 6.4 & 33.9 & 87.8 & 47.0 & 3.5 & 6.3 & 8.2 & 0.93 & 76.1 \\
\hline 15 & $54 / 2013-17$ & 6.3 & 7.1 & 3.0 & 35.2 & 10.8 & 5.9 & 35.1 & 86.8 & 45.8 & 3.5 & 6.8 & 8.3 & 0.93 & 76.8 \\
\hline 16 & $54 / 2013-20$ & 7.3 & 8.5 & 2.8 & 36.8 & 10.9 & 6.3 & 33.6 & 85.8 & 43.6 & 3.3 & 6.8 & 8.6 & 0.88 & 77.7 \\
\hline 17 & \begin{tabular}{|l|}
$57 / 2013-9$ \\
\end{tabular} & 6.7 & 7.6 & 2.7 & 36.4 & 11.1 & 6.4 & 34.4 & 87.2 & 47.3 & 3.5 & 6.8 & 8.7 & 0.92 & 76.0 \\
\hline \multicolumn{2}{|c|}{$\begin{array}{c}\text { Mean of selected } \\
\text { families }\end{array}$} & 7.0 & 8.0 & 2.8 & 36.2 & 11.0 & 6.3 & 34.3 & 86.0 & 47.1 & 3.5 & 6.6 & 8.5 & 0.93 & 77.3 \\
\hline \multicolumn{2}{|c|}{$\begin{array}{c}\text { Mean of } \\
\text { comparisons } \\
\text { (control) }\end{array}$} & 6.8 & 7.8 & 2.8 & 36.6 & 10.6 & 6.1 & 34.2 & 87.5 & 45.5 & 3.6 & 6.7 & 8.5 & 0.9 & 75.5 \\
\hline \multicolumn{2}{|c|}{ SE } & 0.21 & 0.26 & 0.02 & 0.15 & 0.06 & 0.05 & 0.22 & 0.41 & 0.43 & 0.03 & 0.10 & 0.06 & 0.00 & 0.25 \\
\hline \multicolumn{2}{|r|}{ CV \% } & 12.6 & 13.3 & 3.5 & 1.7 & 2.3 & 3.2 & 2.7 & 1.9 & 3.8 & 4.0 & 6.2 & 3.2 & 1.8 & 1.3 \\
\hline \multicolumn{2}{|r|}{$P$ value } & 0.93 & 0.90 & 0.27 & 0.20 & 0.09 & 0.61 & - & - & - & - & - & - & - & - \\
\hline
\end{tabular}

$\mathrm{SE}=$ Standard Error $\quad \mathrm{CV}=$ Coefficient of Variability $\mathrm{P}$ Value $=$ probability value

Application of independent culling levels selection for (increase B) revealed 7 families were selected according to Giza 92 standard characteristics to form nucleolus; these families were homogeneous between them and with control in yield, yield components and fiber properties. Pure seeds of the 7 selected families were massed together to form the breeder's seed stock of Giza 92 cultivar in 2016 season, under name (Giza 92 nucleolus/ 2016), Table 4 presented the 
characters of the selected families. The breeder's seed (nucleolus) was grown in 2016 season in 10 feddans at Sakha farm.

Table 4: Means of yield, yield components and fiber properties of the 7 families selected from increase B in 2015 growing season to form new nucleolus of Giza 92 in 2016 season

\begin{tabular}{|c|c|c|c|c|c|c|c|c|c|c|c|c|c|c|c|}
\hline ì & 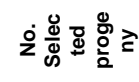 & خ্ّة & 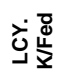 & $\sum_{0}^{\infty}$ & 今̊ & $\frac{\sigma}{\omega}$ & $\frac{\sigma}{3}$ & 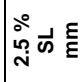 & $\frac{\circ}{3}$ & 巵离 & $\mathbf{z}$ & 亡̊ำ & $\stackrel{0}{+}$ & $\frac{\tilde{c}}{\Sigma}$ & $\stackrel{\circ}{\circ}$ \\
\hline 1 & 5/2013-8 & 5.8 & 6.4 & 2.7 & 35.8 & 11.4 & 6.4 & 35.5 & 87.5 & 48.1 & 3.6 & 6.3 & 8.7 & 0.92 & 78.9 \\
\hline 2 & 5/2013-11 & 7.1 & 7.9 & 2.8 & 35.6 & 11.4 & 6.3 & 34.0 & 87.1 & 50.1 & 3.4 & 6.7 & 8.7 & 0.92 & 77.6 \\
\hline 3 & $26 / 2013-23$ & 6.9 & 7.9 & 3.0 & 36.6 & 11.0 & 6.4 & 36.8 & 86.3 & 45.9 & 3.5 & 6.3 & 8.5 & 0.90 & 77.1 \\
\hline 4 & $52 / 2013-31$ & 6.9 & 8.0 & 2.8 & 36.5 & 11.0 & 6.3 & 34.0 & 83.3 & 46.5 & 3.5 & 6.8 & 8.1 & 0.91 & 77.2 \\
\hline 5 & $53 / 2013-6$ & 7.7 & 8.8 & 2.9 & 36.3 & 10.7 & 6.1 & 34.2 & 89.0 & 49.6 & 3.5 & 6.6 & 8.1 & 0.93 & 76.7 \\
\hline 6 & $54 / 2013-17$ & 6.3 & 7.1 & 3.0 & 35.2 & 10.8 & 5.9 & 35.1 & 86.8 & 45.8 & 3.5 & 6.8 & 8.3 & 0.93 & 76.8 \\
\hline 7 & \begin{tabular}{|c|}
$57 / 2013-9$ \\
\end{tabular} & 6.7 & 7.6 & 2.7 & 36.4 & 11.1 & 6.4 & 34.4 & 87.2 & 47.3 & 3.5 & 6.8 & 8.7 & 0.92 & 76.0 \\
\hline \multicolumn{2}{|c|}{$\begin{array}{l}\text { Mean of selected } \\
\text { progenies }\end{array}$} & 6.8 & 7.7 & 2.8 & 36.1 & 11.1 & 6.3 & 34.9 & 86.7 & 47.6 & 3.5 & 6.6 & 8.4 & 0.92 & 77.2 \\
\hline \multicolumn{2}{|c|}{$\begin{array}{c}\text { Mean of } \\
\text { Comparisons } \\
\text { (control) }\end{array}$} & 6.8 & 7.8 & 2.8 & 36.6 & 10.6 & 6.1 & 34.2 & 87.5 & 45.5 & 3.6 & 6.7 & 8.5 & 0.91 & 75.5 \\
\hline \multirow{2}{*}{\multicolumn{2}{|c|}{$\begin{array}{c}\text { SE } \\
\mathrm{CV} \%\end{array}$}} & 0.23 & 0.29 & 0.05 & 0.20 & 0.10 & 0.07 & 0.39 & 0.66 & 0.65 & 0.02 & 0.09 & 0.10 & 0.00 & 0.34 \\
\hline & & 8.89 & 9.86 & 4.48 & 1.47 & 2.44 & 3.04 & 2.95 & 2.00 & 3.63 & 1.65 & 3.43 & 3.27 & 1.16 & 1.17 \\
\hline
\end{tabular}

$\mathrm{SE}=$ Standard Error $\mathrm{CV}=$ Coefficient of Variability

These results provide good evidence that the pure seed stock released by the cotton breeder would be maintained pure as the stock and exclusively remained under the upper hand of the breeder. Being then the breeder's seed (nucleolus) is further increased to produce foundation seed as a cultivar strain carrying the number of the year of its propagation. On the other hand, deterioration may occur in cotton varieties in general cultivation through contamination by mechanical mixing of different seeds, out crossing with foreign cultivars and offtypes which could result in genetic changes of the cultivar. These results were similar to those obtained by Abdel-Bary and Bisher (1969), Abdel-Al (1976), El-Akkad et al., (1982), El-Kilany and Yossef (1985) and Al-Ameer (2014).

The pure line method in the sense of pedigree selection method for renewing Giza 92 breeder's seed depends on independent culling selection for most traits, this means that the selection technique for producing breeder's seed of Giza 92 cultivar was valid and proved to be effective in holding this cultivar according to the standard type of Giza 92. This may be due to the pedigree selection method used to develop Giza 92 materials during breeding and maintenance period (>15 years) which exhausted the variations due to major genes effects. However minor genes have too small effects to be individually distinguished and segregation occurs at a large number of loci affecting a trait. Thus, the gene frequency changes caused by selection pressure exerted by the breeder, and loss of heterozygosity (due to segregation of heterozygotes remaining even in the most highly bred cultivar), could create some genetic modifications which 
could considered the main reason for appearance of off-type plants in the program. For these reasons continuous selfing and selection procedures carried out every season and considered essential in maintaining program to maintain genetic purity and eliminate any offtype plants from Giza 92 highly bred population.

\section{REFERENCES}

Abd Al-Zaher, G.H. 2004. Maintenance and producing the nucleolus (breeder's seed) of Giza 83 Egyptian Cotton variety, during 20002004 seasons. Egypt. J. Plant Breeding 8: 77-86.

Abdel-Al, M.S.M. 1976. Some aspects of breeding methods for maintaining Egyptian cotton varieties. Ph,D. Thesis Al-Azhar Univ.

Abdel-Bary, A.A. and M.A. Bishr. 1969. Evaluation of new cotton variety Giza 69. Cott. Gr. Rev., 46: 98-104.

Abo-Arab A.R., A.E. Ayoub and A.F. Lasheen. 1995. Maintenance and producing the nucleolus (breeder's seed) of Giza 76 Egyptian cotton variety, during 1990-1992 seasons. Zagazig J. Agric, Res., 22 (2): 399-408.

Al-Ameer, M.A. 2014. Plan for breeding, maintenance and producing the nucleolus (breeder's seed) of Giza 87 Egyptian cotton variety. Egypt. J. Agric. Res., 92 (4), 1341-1355.

Al-Didi, M.A. 1974. Methods of cotton breeding. Egypt. Cott, 62: 49-92.

El-Akkad M.H.; A.F.H. El-Okkia; H. R. El-Hanafi and M.A. Abdel-Dayem 1982. Plan for maintenance and producing the nucleolus (breeder's seed) of Giza 69 Egyptian cotton variety, during 19751979 seasons. Agric. Res. Rev., 60 (9): 111 - 131.

El-Disouqui, A.E. 2001. Maintenance system of Giza 70 Egyptian cotton cultivar. J. Agric., Sci., Mansoura Univ., 26 (4): 1853-1862.

El-Kilany M.A. and S.M. Yousef. 1985. Comparative study on six nuclei seeds of Dandara cotton cultivar and the corresponding farmer seed in general use. Agric. Res. Rev., 63(6): 53-95.

Lasheen, A.F. 1997. Maintenance and producing the nucleolus (breeder's seed) of Giza 75 Egyptian cotton variety. Menofiya J. Agric. Res., 22 (5): 1279-1290.

Lewis, C.F. 1970. Concepts of varietal maintenance in cotton. Cott. Gr. Rev., 47: 272-284.

Mohamed, A.A. 2013. Maintenance and producing the nucleolus (breeder's seed) of Giza 90 Egyptian cotton variety, during 20092012 seasons. J. Agric. Res. Kafr El-Sheikh Univ., 39 :(1): 79-91.

Nagib, M.A.A and G.M. Hemida. 2001. Some aspects on cotton variety renewal and maintenance scheme of Giza 80 . Minia J., of Agric. Res, of Develop. 21 (1): 67-75.

Riggs, T. J. 1967. Response to model selection in Upland cotton in northern and eastern Uganda. Cott. Gr.Rev., 44: 176-183.

Turner, J. H. (1963). Breeding method used in maintenance and improvement of Acala 4-42 variety of cotton. U.S. Dept.Ar. ARS Cotton Res.Sta. Shafter. Calif., 67-75. 
Walker, J. T. 1964. Model selection in Upland cotton. Heredity, 19: 559583.

Ware, J.O. 1959. Plan for breeding, maintenance and propagation of Egyptian cotton varieties. A report submitted to the ministry of Agric., Egypt.

Younis F,G., E.M. Ghoneim and M.O.Ismail, 1993. Producing the nucleolus (breeder's seed) of Dandara Eyptian cotton variety, during 1988-1991 seasons. Egypt. Jape. Sci., 8 (2): 238-248.

\section{المحافظة و انتاج النوية (بذرة المربي) لصنف القطن المصري جيزة 92

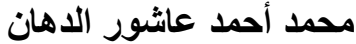

$$
\text { معهز بحوث القطن - مركز البحوث الزراعية ـ- مصر }
$$

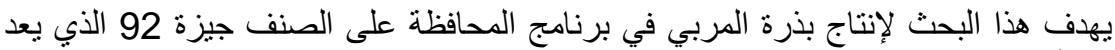

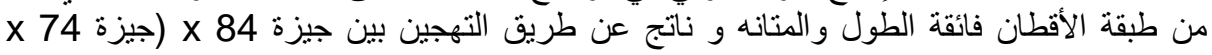

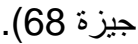

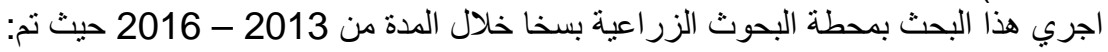

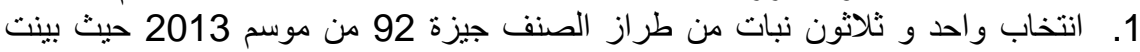

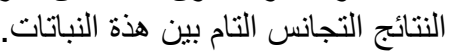
2. في موسم 2014 تم زر اعة نسل هذه النباتاتات مكون إكثار ارت النات (أ) و في نهاية الموسم و

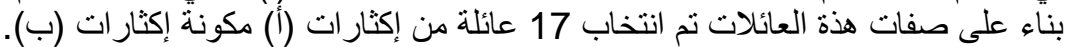

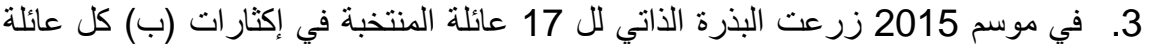

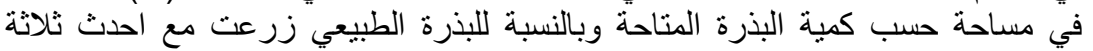

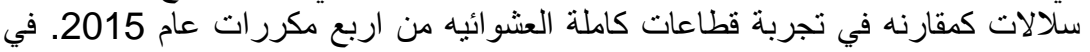

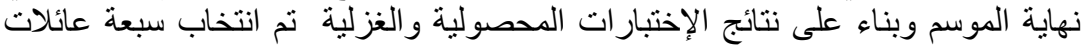
نموذجية تمثل الصنف جيزة 92 في صفاه

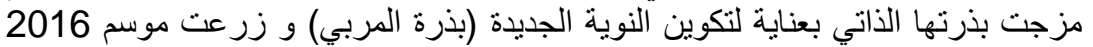

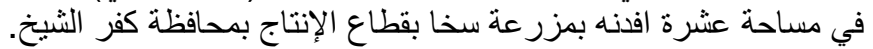

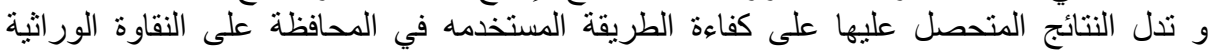
للصنف جيزة 92 و ذللك بمزج بذرة الإن الانسال السبعه المنتخبه معا.

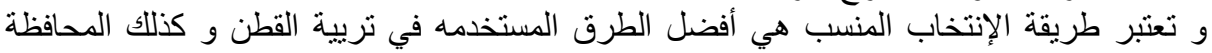

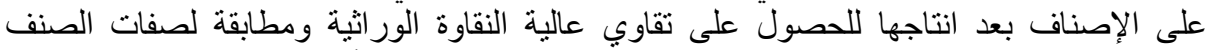

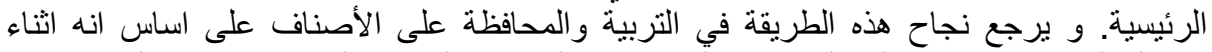

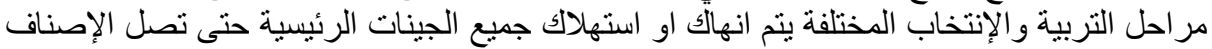

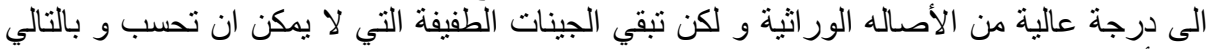

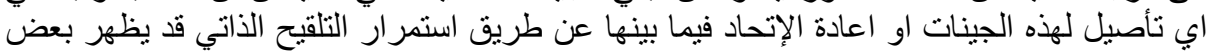

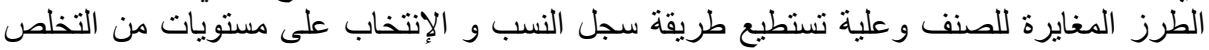

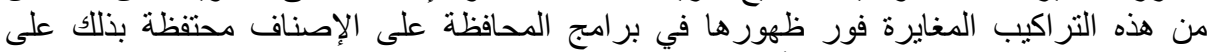

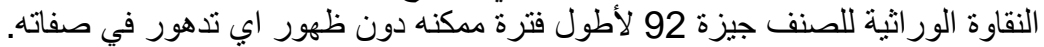

